\title{
NILPOTENT LIE ALGEBRAS OF CLASS 4 WITH THE DERIVED SUBALGEBRA OF DIMENSION 3
}

\author{
FARANGIS JOHARI, PEYMAN NIROOMAND, AND MOHSEN PARVIZI
}

\begin{abstract}
The paper is devoted to give a full classification of all finite dimensional nilpotent Lie algebras $L$ of class 4 such that $\operatorname{dim} L^{2}=3$. Moreover, we classify the capable ones.
\end{abstract}

\section{INTRODUCTION}

It is well known that the classification of nilpotent Lie algebras is a classical problem. Several classifications of nilpotent Lie algebras of dimension at most 7 over various ground fields are available in the literature (See 4, 6, 7]). It is not easy to classify nilpotent Lie algebras with an arbitrary dimension. Hence we are interested to classify nilpotent Lie algebras by focusing on some other aspects rather than the dimension. For a given Lie algebra $L$ with $\operatorname{dim} L^{2}=1$, the structure of $L$ is given in 10. When $\operatorname{dim} L^{2}=2$, we gave the structure of $L$ when $L$ is of class 3 and with some restrictions for class 2 in [13. The purpose of this paper is to describe a classification of all nilpotent Lie algebras of class 4 with the derived subalgebra of dimension 3. Moreover, in this class, we classify which ones are capable.

\section{Preliminaries}

This section is devoted to give some elementary and known results that will be needed for the next investigations. All Lie algebras in this paper are finite dimensional over any arbitrary field.

First we recall the concept of a central product of two Lie algebras $A$ and $B$.

Definition 2.1. A Lie algebra $L$ is a central product of $A$ and $B$, if $L=A+B$, where $A$ and $B$ are ideals of $L$ such that $[A, B]=0$ and $A \cap B \subseteq Z(L)$. We denote the central product of two Lie algebras $A$ and $B$ by $A \dot{+} B$.

The following lemma emphasizes the Heisenberg Lie algebras are in fact central products some of their ideals.

Lemma 2.2. 8, Lemma 3.3] Let $L$ be a Heisenberg Lie algebra of dimension $2 m+1$. Then $L$ is a central product of its ideals $B_{j}$ for all $j, 1 \leq j \leq m$ such that each $B_{j}$ is the Heisenberg Lie algebra of dimension 3.

A Lie algebra $L$ is called capable provided that $L \cong H / Z(H)$ for some Lie algebra $H$. The notion of the epicenter $Z^{*}(L)$ for a Lie algebra $L$ was defined in [14. It is shown that $L$ is capable if and only if $Z^{*}(L)=0$. Another notion having a relation to the capability is the concept of the exterior square of Lie algebras, $L \wedge L$, which

Date: July 31, 2018.

Mathematics Subject Classification 2010. Primary 17B30; Secondary 17B05, 17B99. 
was introduced in [5]. Our approach is on the concept of the exterior center $Z^{\wedge}(L)$, the set of all elements $l$ of $L$ for which $l \wedge l^{\prime}=0_{L \wedge L}$ for all $l^{\prime} \in L$. Niroomand et al. in [11] showed $Z^{\wedge}(L)=Z^{*}(L)$ for any finite dimensional Lie algebra $L$.

It is not an easy matter to determine the capability of a central product of Lie algebras in general, but the next result gives the answer to this question in a particular case.

Proposition 2.3. 12, Proposition 2.2] Let $L$ be a Lie algebra such that $L=A \dot{+} B$ with $A^{2} \cap B^{2} \neq 0$. Then $A^{2} \cap B^{2} \subseteq Z^{\wedge}(L)$. Moreover, $L$ is non-capable.

Let $c l(L)$ denote the nilpotency class of a Lie algebra $L$. The following theorem gives the classification of all capable nilpotent Lie algebras of class 3 with the derived subalgebra of dimension 2.

Theorem 2.4. [13, Theorem 5.3] Let $L$ be an $n$-dimensional Lie algebra such that $c l(L)=3$ and $\operatorname{dim} L^{2}=2$. Then $L$ is capable if and only if $L \cong L_{4,3} \oplus A(n-4)$ or $L \cong L_{5,5} \oplus A(n-5)$.

From [7, the only Lie algebra of maximal class of dimension 4 is isomorphic to

$$
L_{4,3}=\left\langle x_{1}, \ldots, x_{4} \mid\left[x_{1}, x_{2}\right]=x_{3},\left[x_{1}, x_{3}\right]=x_{4}\right\rangle,
$$

and there are two Lie algebras of maximal class of dimension 5 that are isomorphic to

$$
L_{5,6}=\left\langle x_{1}, \ldots, x_{5} \mid\left[x_{1}, x_{2}\right]=x_{3},\left[x_{1}, x_{3}\right]=x_{4},\left[x_{1}, x_{4}\right]=x_{5},\left[x_{2}, x_{3}\right]=x_{5}\right\rangle
$$

and

$$
L_{5,7}=\left\langle x_{1}, \ldots, x_{5} \mid\left[x_{1}, x_{2}\right]=x_{3},\left[x_{1}, x_{3}\right]=x_{4},\left[x_{1}, x_{4}\right]=x_{5}\right\rangle,
$$

respectively.

We say a Lie algebra $L$ is a semidirect sum of an ideal $I$ by a subalgebra $K$ if $L=I+K, I \cap K=0$. The semidirect sum of an ideal $I$ by a subalgebra $K$ is denoted by $K \ltimes I$.

Lemma 2.5. [13, Lemma 4.1] Let $L$ be a 5-dimensional nilpotent stem Lie algebra of class 3 and $\operatorname{dim} L^{2}=2$. Then

$$
L \cong L_{5,5}=\left\langle x_{1}, \ldots, x_{5} \mid\left[x_{1}, x_{2}\right]=x_{3},\left[x_{1}, x_{3}\right]=x_{5},\left[x_{2}, x_{4}\right]=x_{5}\right\rangle .
$$

Moreover, $L_{5,5}=I \rtimes\left\langle x_{4}\right\rangle$ in which

$I=\left\langle x_{1}, x_{2}, x_{3}, x_{5} \mid\left[x_{1}, x_{2}\right]=x_{3},\left[x_{1}, x_{3}\right]=x_{5}\right\rangle \cong L_{4,3}$, and $\left[I,\left\langle x_{4}\right\rangle\right]=\left\langle x_{5}\right\rangle=Z\left(L_{5,5}\right)$.

Lemma 2.6. Let $L$ be a 6-dimensional nilpotent stem Lie algebra of class 4 and $\operatorname{dim} L^{2}=3$. Then $L$ is isomorphic to one of the Lie algebras listed below.

(1) $L_{6,11}=\left\langle x_{1}, \ldots, x_{6}\right|\left[x_{1}, x_{2}\right]=x_{3},\left[x_{1}, x_{3}\right]=x_{4},\left[x_{1}, x_{4}\right]=\left[x_{2}, x_{3}\right]=\left[x_{2}, x_{5}\right]=$ $\left.x_{6}\right\rangle=I_{1} \rtimes\left\langle x_{5}\right\rangle$, in which $I_{1}=\left\langle x_{1}, x_{2}, x_{3}, x_{4}, x_{6}\right|\left[x_{1}, x_{2}\right]=x_{3},\left[x_{1}, x_{3}\right]=$ $\left.x_{4},\left[x_{1}, x_{4}\right]=\left[x_{2}, x_{3}\right]=x_{6}\right\rangle \cong L_{5,6}$ and $\left[I,\left\langle x_{5}\right\rangle\right]=\left\langle x_{6}\right\rangle=Z\left(I_{1}\right)$.

(2) $L_{6,12}=\left\langle x_{1}, \ldots, x_{6} \mid\left[x_{1}, x_{2}\right]=x_{3},\left[x_{1}, x_{3}\right]=x_{4},\left[x_{1}, x_{4}\right]=\left[x_{2}, x_{5}\right]=x_{6}\right\rangle=$ $I_{2} \rtimes\left\langle x_{5}\right\rangle$, in which $I_{2}=\left\langle x_{1}, x_{2}, x_{3}, x_{4}, x_{6}\right|\left[x_{1}, x_{2}\right]=x_{3},\left[x_{1}, x_{3}\right]=x_{4},\left[x_{1}, x_{4}\right]=$ $\left.x_{6}\right\rangle \cong L_{5,7}$ and $\left[I_{2},\left\langle x_{5}\right\rangle\right]=\left\langle x_{6}\right\rangle=Z\left(I_{2}\right)$.

(3) $L_{6,13}=\left\langle x_{1}, \ldots, x_{6}\right|\left[x_{1}, x_{2}\right]=x_{3},\left[x_{1}, x_{3}\right]=\left[x_{2}, x_{4}\right]=x_{5},\left[x_{1}, x_{5}\right]=\left[x_{3}, x_{4}\right]=$ $\left.x_{6}\right\rangle=I_{3} \rtimes\left\langle x_{4}\right\rangle$, in which $I_{3}=\left\langle x_{1}, x_{2}, x_{3}, x_{5}, x_{6}\right|\left[x_{1}, x_{2}\right]=x_{3},\left[x_{1}, x_{3}\right]=$ $\left.x_{5},\left[x_{1}, x_{5}\right]=x_{6}\right\rangle \cong L_{5,7}$ and $\left[I_{3},\left\langle x_{4}\right\rangle\right]=\left\langle x_{5}, x_{6}\right\rangle$. 
Proof. By looking at the classification of nilpotent Lie algebras of dimension at most 6 in [4, 7], we get $L \cong L_{6,11}, L \cong L_{6,12}$ or $L \cong L_{6,13}$. Let $L \cong L_{6,11}$. It is easy to check that $L_{6,11}=I_{1} \rtimes\left\langle x_{5}\right\rangle$, in which $I_{1}=\left\langle x_{1}, x_{2}, x_{3}, x_{4}, x_{6}\right|\left[x_{1}, x_{2}\right]=$ $\left.x_{3},\left[x_{1}, x_{3}\right]=x_{4},\left[x_{1}, x_{4}\right]=\left[x_{2}, x_{3}\right]=x_{6}\right\rangle \cong L_{5,6}$ and $\left[I,\left\langle x_{5}\right\rangle\right]=\left\langle x_{6}\right\rangle=Z\left(L_{5,6}\right)$. Similarly, we can see that $L_{6,12}=\left\langle x_{1}, \ldots, x_{6}\right|\left[x_{1}, x_{2}\right]=x_{3},\left[x_{1}, x_{3}\right]=x_{4},\left[x_{1}, x_{4}\right]=$ $\left.\left[x_{2}, x_{5}\right]=x_{6}\right\rangle=I_{2} \rtimes\left\langle x_{5}\right\rangle$, in which $I_{2}=\left\langle x_{1}, x_{2}, x_{3}, x_{4}, x_{6}\right|\left[x_{1}, x_{2}\right]=x_{3},\left[x_{1}, x_{3}\right]=$ $\left.x_{4},\left[x_{1}, x_{4}\right]=x_{6}\right\rangle \cong L_{5,7}$ and $\left[I_{2},\left\langle x_{5}\right\rangle\right]=\left\langle x_{6}\right\rangle=Z\left(L_{5,7}\right)$. Now, let $L \cong L_{6,13}$. Clearly $Z(L)=\left\langle x_{6}\right\rangle$ and $L_{6,13}=I_{3}+\left\langle x_{4}\right\rangle$, where $I_{3}=\left\langle x_{1}, x_{2}, x_{3}, x_{5}, x_{6}\right|\left[x_{1}, x_{2}\right]=$ $\left.x_{3},\left[x_{1}, x_{3}\right]=x_{5},\left[x_{1}, x_{5}\right]=x_{6}\right\rangle \cong L_{5,7}$ and $\left[I_{3},\left\langle x_{4}\right\rangle\right]=\left\langle x_{5}, x_{6}\right\rangle$, as required.

We need the following lemma.

Lemma 2.7. 15, Lemma 1] Let $L$ be a nilpotent Lie algebra and $H$ be a subalgebra of $L$ such that $L^{2}=H^{2}+L^{3}$. Then $L^{i}=H^{i}$ for all $i \geq 2$. Moreover, $H$ is an ideal of $L$.

\section{MAin RESUlts}

We are going to give the structure of all nilpotent Lie algebras of class 4 with the derived subalgebra of dimension 3. Moreover, we determine which one of these are capable.

The next two results which are stated for Lie algebras have a group theoretical reason for $p$-groups in [2, Lemma 2.3 and Theorem 2.4]. Here, we give a proof for them.

Lemma 3.1. Let $L$ be an $n$-dimensional nilpotent Lie algebra of class $c$ such that $\operatorname{dim} L^{2}=c-1$ and $I$ be an ideal of dimension $i(0 \leq i \leq c-1)$ contained in $L^{2}$. Then $I=L^{c-i+1}$.

Proof. Clearly, $\operatorname{dim} L^{j}=c-j+1$, where $1 \leq j \leq c$. We proceed by induction on $c-i+1$. If $i=c-1$, the result follows easily. Let $c-i>1$ and $M / I$ be an ideal of dimension 1 such that $M / I \subseteq(L / I)^{2} \cap Z(L / I)$. So $M$ is an $(i+1)$-dimensional ideal of $L$ such that $I \varsubsetneqq M \subseteq L^{2}$. By using the induction hypothesis, $M=L^{c-i}$. Since $M / I \subseteq Z(L / I)$, we have $L^{c-i+1}=\left[L, L^{c-i}\right]=[L, M] \subseteq I$. Now, both $I$ and $L^{c-i+1}$ are of dimension $i$, and hence $I=L^{c-i+1}$. The result follows.

Recall that an $n$-dimensional nilpotent Lie algebra $L$ is said to be nilpotent of maximal class if $\operatorname{cl}(L)=n-1$. For a maximal class Lie algebra $L$, we have $\operatorname{dim}\left(L / L^{2}\right)=2, Z_{i}(L)=L^{n-i}$ and $\operatorname{dim}\left(L^{j} / L^{j+1}\right)=1$ for all $i, j, 0 \leq i \leq n-1$ and $2 \leq j \leq n-1$ (see [3] for more information).

Proposition 3.2. Let $L$ be an $n$-dimensional Lie algebra of class $c$ such that $\operatorname{dim} L^{2}=c-1$. Then $L^{2} \cap Z_{i}(L)=L^{c-i+1}$ for all $i, 0 \leq i \leq c-1$.

Proof. By contrary, let $L^{c-i+1} \varsubsetneqq L^{2} \cap Z_{i}(L)$. Since $\operatorname{dim} L^{c-i+1}=i$, we get $k=$ $\operatorname{dim}\left(L^{2} \cap Z_{i}(L)\right) \geq i+1$. Lemma 3.1 implies $L^{2} \cap Z_{i}(L)=L^{c-k+1} \subseteq L^{c-i+1}$. Since $\operatorname{dim} L^{c-i+1}=i$, we have a contradiction. This completes the proof.

Recall that from [9] a Lie algebra $S$ is called a stem Lie algebra if $Z(S) \subseteq S^{2}$. Now, we are able to prove the following result which is useful in the rest.

Proposition 3.3. Let $L$ be an $n$-dimensional Lie algebra of class $c$ such that $\operatorname{dim} L^{2}=c-1$. Then $L$ is stem if and only if $Z(L)=L^{c} \cong A(1)$. 
Proof. Let $L$ be stem. Then by Proposition 3.2 we have

$$
L^{c} \subseteq Z(L) \subseteq L^{2} \cap Z(L)=L^{c} .
$$

This implies $\operatorname{dim} Z(L)=\operatorname{dim} L^{c}=1$, as required.

Theorem 3.4. Let $L$ be an $n$-dimensional nilpotent stem Lie algebra of class 4 $(n \geq 6)$ and $\operatorname{dim} L^{2}=3$ such that $L=I+K$, where $K$ is a subalgebra of $L$ and $I$ is a maximal class subalgebra of dimension 5 such that $[I, K] \subseteq Z(I)=Z(L)$. Then

(i) If $K$ is a non-trivial abelian Lie algebra such that $K \cap I=0$, then $[I, K]=$ $Z(L)$ and $K \cong A(1)$. Moreover, $L=I \rtimes K$ and $L$ is isomorphic to $L_{6,11}$ or $L_{6,12}$.

(ii) If $K$ is a Heisenberg Lie algebra and $I \cap K=Z(L) \cong A(1)$, then $L$ is isomorphic to one of the following Lie algebras.

(a) $L \cong I+K \cong\left\langle x_{1}, \ldots, x_{5}, a, b\right|\left[x_{1}, x_{i}\right]=x_{i+1},\left[x_{2}, x_{3}\right]=[a, b]=x_{5}, 1 \leq$ $i \leq 4\rangle$, where $I \cong L_{5,6}$ and $K \cong H(1)$.

(b) $L \cong I+K \cong\left\langle x_{1}, \ldots, x_{5}, a, b \mid\left[x_{1}, x_{i}\right]=x_{i+1},[a, b]=x_{5}, 1 \leq i \leq 4\right\rangle$, where $I \cong L_{5,7}$ and $K \cong H(1)$.

(c) $L \cong I \dot{+} K \cong I_{1} \dot{+} K_{1}$, where $I_{1} \cong\left\langle x_{1}, \ldots, x_{5}, a, b\right|\left[x_{1}, x_{i}\right]=x_{i+1},\left[x_{2}, x_{3}\right]$ $\left.=[a, b]=x_{5}, 1 \leq i \leq 4\right\rangle, K_{1} \cong H(m-1)$ and $n=2 m+5$ for all $m \geq 2$.

(d) $L \cong I \dot{+} K \cong I_{2} \dot{+} K_{2}$, where $I_{2} \cong\left\langle x_{1}, \ldots, x_{5}, a, b\right|\left[x_{1}, x_{i}\right]=x_{i+1},[a, b]=$ $\left.x_{5}, 1 \leq i \leq 4\right\rangle, K_{2} \cong H(m-1)$ and $n=2 m+5$ for all $m \geq 2$.

(iii) If $A(1) \cong I \cap K=Z(L)=K^{2} \neq Z(K)$, then $L \cong(I \rtimes A) \dot{+} K$, where $K \cong H(m)$ and $A \cong A(1),[I, A]=Z(L)=Z(I)=K^{2}$ and $n=2 m+6$. Moreover, $I \rtimes A \cong L_{6,11}$ or $I \rtimes A \cong L_{6,12}$.

Proof. (i) We have $[I, K] \subseteq Z(I)=Z(L)$, so $I$ is an ideal of $L$ and $I \cong L_{5,6}$ or $I \cong L_{5,7}$. We know that $I$ is a Lie algebra of maximal class of dimension 4 so $Z(I)=Z(L)=I^{4}$. We also have $\operatorname{dim} L^{2}=\operatorname{dim} I^{2}=3$. Therefore $L^{2}=I^{2}$. Since $K \cap I=0$, we obtain $\operatorname{dim} K=\operatorname{dim} L-\operatorname{dim} I=n-5$ and so $L=I \rtimes K$, in which $K \cong A(n-5)$. We claim that $[I, K]=Z(I)$. By contrary, assume that $[I, K]=0$. Since $K$ is abelian, we have $K \subseteq Z(I)=Z(L) \subseteq I$. Now $I \cap K \neq 0$, so we have a contradiction. Thus $[I, K]=Z(I)=Z(L)$.

We claim that $K \cong A(1)$.

First assume that $n=6$. Lemma 2.6 implies $L \cong I_{1} \rtimes K \cong L_{6,11}$ or $L \cong I_{2} \rtimes K \cong L_{6,12}$, in which $I_{1} \cong L_{5,6}, I_{2} \cong L_{5,7},\left[I_{1}, K\right]=Z(L)$ and $\left[I_{2}, K\right]=Z(L)$.

Now, let $n \geq 7$ and $K=\bigoplus_{i=1}^{n-5}\left\langle z_{i}\right\rangle$. In this case we show that $K \cap I \neq 0$, which is impossible, so this case cannot occur. By using the Jacobi identity, for all $i, 1 \leq i \leq n-5$, we have

$$
\left[z_{i}, x_{3}\right]=\left[z_{i},\left[x_{1}, x_{2}\right]\right]=\left[z_{i}, x_{1}, x_{2}\right]+\left[x_{2}, z_{i}, x_{1}\right]=0
$$

and

$$
\left[z_{i}, x_{4}\right]=\left[z_{i},\left[x_{1}, x_{3}\right]\right]=\left[z_{i}, x_{1}, x_{3}\right]+\left[x_{3}, z_{i}, x_{1}\right]=0
$$

since $\left[z_{i}, x_{1}\right],\left[z_{i}, x_{3}\right]$ and $\left[x_{2}, z_{i}\right]$ are central. Thus $\left[z_{i}, x_{3}\right]=\left[z_{i}, x_{4}\right]=0$ for all $i, 1 \leq i \leq n-5$. Now, let $\left[z_{i}, x_{1}\right]=\alpha_{i} x_{6}$ and $\alpha_{i} \neq 0$ for all $i$, $1 \leq i \leq n-5$. Putting $z_{i}^{\prime}=z_{i}+\alpha_{i} x_{4}$, we have $\left[z_{i}^{\prime}, x_{1}\right]=\left[z_{i}+\alpha_{i} x_{4}, x_{1}\right]=$ $\left[z_{i}, x_{1}\right]+\alpha_{i}\left[x_{4}, x_{1}\right]=\alpha_{i} x_{6}-\alpha_{i} x_{6}=0$. Also we obtain $\left[z_{i}^{\prime}, x_{4}\right]=0$. Thus $\left[z_{i}^{\prime}, x_{1}\right]=\left[z_{i}^{\prime}, x_{3}\right]=\left[z_{i}^{\prime}, x_{4}\right]=0$ for all $i, 1 \leq i \leq n-5$. Now let $\left[z_{i}^{\prime}, x_{2}\right]=\alpha x_{6}$ 
and $\left[z_{j}^{\prime}, x_{2}\right]=\beta x_{6}$, in which $\alpha \neq 0$ and $\beta \neq 0$ for some fixed $i, j$, with $i \neq j$. Put $d_{i j}=\beta z_{i}^{\prime}-\alpha z_{j}^{\prime}$. We have

$$
\left[d_{i j}, x_{2}\right]=\left[\beta z_{i}^{\prime}-\alpha z_{j}^{\prime}, x_{2}\right]=\beta \alpha x_{6}-\beta \alpha x_{6}=0
$$

and so $\left[d_{i j}, x_{2}\right]=0$. On the other hand, $\left[d_{i j}, x_{1}\right]=\left[d_{i j}, x_{2}\right]=\left[d_{i j}, x_{3}\right]=$ $\left[d_{i j}, x_{4}\right]=0$. Therefore $\left[d_{i j}, I\right]=0$ and hence $d_{i j} \in Z(L)=Z(I)=\left\langle x_{6}\right\rangle$. Since

$$
\begin{aligned}
& d_{i j}=\beta z_{i}^{\prime}-\alpha z_{j}^{\prime}=\beta\left(z_{i}-\alpha_{i} x_{4}\right)-\alpha\left(z_{j}-\alpha_{j} x_{4}\right) \\
& =\beta z_{i}-\alpha z_{j}+\left(\alpha \alpha_{j}-\beta \alpha_{i}\right) x_{4} \in Z(I),
\end{aligned}
$$

$0 \neq \beta z_{i}-\alpha z_{j} \in K \cap I=0$, which is a contradiction. Thus $n=5$, $K \cong A(1), L=I \rtimes\left\langle z_{1}\right\rangle$ and $\left[x_{2}, z_{1}\right]=x_{6}$, as required. Now, considering the classification of nilpotent Lie algebras of dimension 6 with $\operatorname{dim} L^{2}=3$ which is given in [7, using Lemma 2.6 and our assumption, we should have $L \cong L_{6,11}$ or $L \cong L_{6,12}$.

(ii) Since $I \cap K=Z(L) \cong A(1)$, we have $I \cap K=Z(L)=Z(K)$ and $\operatorname{dim}(K)=$ $\operatorname{dim}(L)-\operatorname{dim}(I)+\operatorname{dim}(I \cap K)=n-5+1=n-4$. Now since $2 m+1=$ $\operatorname{dim}(K)=n-4$, we have $n=2 m+5$. We are going to show that $[I, K]=0$. In fact, we show that there exists $I_{1} \cong L_{5,6}$ or $I_{1} \cong L_{5,7}$ and $K_{2} \cong H(m)$ with $\left[I_{1}, K_{2}\right]=0$ and $L=I_{1}+K_{2}$. First let $m=1$. We have $\operatorname{dim} L=6$ and $K=\left\langle x, y, x_{6} \mid[x, y]=x_{6}\right\rangle$, since $K \cong H(1)$. By using the Jacobi identity, we have

$$
\begin{aligned}
& {\left[x, x_{3}\right]=\left[x,\left[x_{1}, x_{2}\right]\right]=\left[x, x_{1}, x_{2}\right]+\left[x_{2}, x, x_{1}\right]=0,} \\
& {\left[x, x_{4}\right]=\left[x,\left[x_{1}, x_{3}\right]\right]=\left[x, x_{1}, x_{3}\right]+\left[x_{3}, x, x_{1}\right]=0,} \\
& {\left[y, x_{3}\right]=\left[y,\left[x_{1}, x_{2}\right]\right]=\left[y, x_{1}, x_{2}\right]+\left[x_{2}, y, x_{1}\right]=0}
\end{aligned}
$$

and

$$
\left[y, x_{4}\right]=\left[y,\left[x_{1}, x_{3}\right]\right]=\left[y, x_{1}, x_{3}\right]+\left[x_{3}, y, x_{1}\right]=0 .
$$

Thus $\left[x, x_{3}\right]=\left[x, x_{4}\right]=\left[y, x_{3}\right]=\left[y, x_{4}\right]=0$. Now, let $\left[x, x_{1}\right]=\alpha x_{6}$ and $\left[y, x_{1}\right]=\beta x_{6}$ for some scalars $\alpha$ and $\beta$. Then by taking $x^{\prime}=x+\alpha x_{4}$ and $y^{\prime}=y+\beta x_{4}$, we have $\left[x^{\prime}, x_{1}\right]=\left[y^{\prime}, x_{1}\right]=0$. Without loss of generality, let $\left[y^{\prime}, x_{2}\right]=0$ and assume that $\left[x^{\prime}, x_{2}\right]=\eta x_{6}$ for a scalar $\eta$. By taking $x_{2}^{\prime}=x_{2}-\eta y^{\prime}$, we have $\left[x^{\prime}, x_{2}^{\prime}\right]=0$. Thus $L=I \dot{+} K$, where $K \cong H(1)$ and $I$ is a maximal class Lie algebra of dimension 5. Thus $L \cong\left\langle x_{1}, \ldots, x_{5}, a, b \mid\left[x_{1}, x_{i}\right]=x_{i+1},\left[x_{2}, x_{3}\right]=[a, b]=x_{5}, 1 \leq i \leq 4\right\rangle$ or $L \cong\left\langle x_{1}, \ldots, x_{5}, a, b \mid\left[x_{1}, x_{i}\right]=x_{i+1},[a, b]=x_{5}, 1 \leq i \leq 4\right\rangle$. It completes cases $(a)$ and $(b)$ of $(i i)$.

Now, let $m \geq 2$ and $H(m)=\left\langle a_{1}, b_{1}, \ldots, a_{m}, b_{m}, z \mid\left[a_{l}, b_{l}\right]=z, 1 \leq l \leq m\right\rangle$. Lemma 2.2 implies that $H(m)=T_{1} \dot{+} \ldots \dot{+} T_{m}$, in which $T_{i} \cong H(1)$ for all $i, 1 \leq i \leq m$. With the same procedure used in case $(i)$ and changing the variables we can see that $\left[T_{i}, I\right]=0$ for all $i, 1 \leq i \leq m$. So $[I, K]=0$ and hence $L=I+K$. Since $m \geq 2$, we have $L=\left(I+T_{1}\right) \dot{+}\left(T_{2} \dot{+} \ldots \dot{+} T_{m}\right)$ such that $I+T_{1} \cong\left\langle x_{1}, \ldots, x_{5}, a, b\right|\left[x_{1}, x_{i}\right]=x_{i+1},\left[x_{2}, x_{3}\right]=[a, b]=x_{5}, 1 \leq$ $i \leq 4\rangle, K_{1} \cong H(m-1)$ or $I+T_{1} \cong\left\langle x_{1}, \ldots, x_{5}, a, b\right|\left[x_{1}, x_{i}\right]=x_{i+1},[a, b]=$ $\left.x_{5}, 1 \leq i \leq 4\right\rangle$ and $T_{2} \dot{+} \ldots \dot{+} T_{m} \cong H(m-1)$. It completes cases $(c)$ and (d) of $(i i)$. 
(iii) Since $I \cap K=K^{2}=Z(L)=Z(I) \cong A(1), \operatorname{dim}(K)=\operatorname{dim}(L)-\operatorname{dim}(I)+$ $\operatorname{dim}(I \cap K)=n-5+1=n-4$. We know that $\operatorname{dim} K^{2}=1$, so [11, Theorem 3.6] implies $K \cong K_{1} \oplus A$, in which $K_{1} \cong H(m)$ and $A \cong A(n-2 m-5)$. Since $A \neq 0$, we have $n \neq 2 m-5$. Thus $L=I+\left(K_{1} \oplus A\right)$ such that $[I, K] \subseteq Z(L)=Z(I)$. We are going to show that $A \cong A(1),\left[I, K_{1}\right]=0$ and $[I, A]=Z(I)=Z(L)$. Similar to part $(i i)$, we can see that $\left[I, K_{1}\right]=0$. We claim that $[I, A] \neq 0$. By contrary, let $\left[A, K_{1}\right]=[I, A]=0$, so $A \subseteq$ $Z(L)=Z(I)$. Since $A \cap I=0$, we have $A=0$, which is a contradiction. So we have $[I, A]=Z(L)$. We claim that $\operatorname{dim} A=1$. Let $\operatorname{dim} A \geq 2$. Similar to the proof of part $(i)$, we have $\left[a_{1}, x_{1}\right]=\left[a_{2}, x_{1}\right]=\left[a_{1}, x_{3}\right]=$ $\left[a_{2}, x_{3}\right]=\left[a_{1}, x_{4}\right]=\left[a_{2}, x_{4}\right]=0$ where $a_{1}, a_{2} \in A$ and $a_{1} \neq a_{2}$. Now let $\left[a_{1}, x_{2}\right]=\alpha x_{5}$ and $\left[a_{2}, x_{2}\right]=\beta x_{5}$ for some non-zero scalars $\alpha$ and $\beta$. Putting $a_{1}^{\prime}=\beta a_{1}-\alpha a_{2}$. We have $\left[a_{1}^{\prime}, x_{2}\right]=\left[\beta a_{1}-\alpha a_{2}, x_{2}\right]=\beta \alpha x_{5}-\beta \alpha x_{5}=0$ and so $\left[a_{1}^{\prime}, x_{2}\right]=0$. Hence $\left[a_{1}^{\prime}, x_{1}\right]=\left[a_{1}^{\prime}, x_{2}\right]=\left[a_{1}^{\prime}, x_{3}\right]=\left[a_{1}^{\prime}, x_{4}\right]=0$. Therefore $\left[a_{1}^{\prime}, I\right]=0$ and hence $a_{1}^{\prime} \in Z(L)=Z(I)=\left\langle x_{5}\right\rangle=K_{1}^{2}$. So $a_{1}^{\prime} \in K_{1}$ and since $K_{1} \cap A=0$, we have a contradiction. Hence $A \cong A(1)$ and so $n=2 m+6$. Thus $L=(I \rtimes A) \dot{+} K_{1}$ such that $[I, A]=Z(L)=Z(I)$. By using part $(i)$, we have $I \rtimes A \cong L_{6,11}$ or $I \rtimes A \cong L_{6,12}$. The proof of case (iii) is completed.

Proposition 3.5. Let $L$ be an $n$-dimensional nilpotent stem Lie algebra of class 4 $(n \geq 7)$ and $\operatorname{dim} L^{2}=3$ such that $L=I+K$, where $I$ and $K$ are two subalgebras of $L, I \cong L_{6,13}$ and $[I, K] \subseteq Z(I)=Z(L) \cong A(1)$. Then

(i) If $K$ is a non-trivial abelian Lie algebra such that $K \cap I=0$, then $[I, K]=$ $Z(L)$ and $K \cong A(1)$. Moreover, $L=I \rtimes K \cong\left\langle x_{1}, \ldots, x_{6}, x_{7}\right|\left[x_{1}, x_{2}\right]=$ $\left.x_{3},\left[x_{1}, x_{3}\right]=\left[x_{2}, x_{4}\right]=x_{5},\left[x_{1}, x_{5}\right]=\left[x_{3}, x_{4}\right]=\left[x_{2}, x_{7}\right]=\left[x_{4}, x_{7}\right]=x_{6}\right\rangle$.

(ii) If $K$ is a Heisenberg Lie algebra and $I \cap K=Z(L) \cong A(1)$, then $L$ is isomorphic to one of the following Lie algebras.

(a) $L \cong I+K \cong\left\langle x_{1}, \ldots, x_{6}, a, b\right|\left[x_{1}, x_{2}\right]=x_{3},\left[x_{1}, x_{3}\right]=\left[x_{2}, x_{4}\right]=$ $\left.x_{5},\left[x_{1}, x_{5}\right]=\left[x_{3}, x_{4}\right]=[a, b]=x_{6}\right\rangle$, where $K \cong H(1)$.

(b) $L \cong I \dot{+} K \cong I_{1} \dot{+} I_{2}$, where $I_{1} \cong\left\langle x_{1}, \ldots, x_{6}, a, b\right|\left[x_{1}, x_{2}\right]=x_{3},\left[x_{1}, x_{3}\right]=$ $\left.\left[x_{2}, x_{4}\right]=x_{5},\left[x_{1}, x_{5}\right]=\left[x_{3}, x_{4}\right]=[a, b]=x_{6}\right\rangle, I_{2} \cong H(m-1)$ and $n=2 m+6$ for all $m \geq 2$.

(iii) If $A(1) \cong I \cap K=Z(L)=K^{2} \neq Z(K)$, then $L=(I \rtimes A)+K$, where $K \cong$ $H(m), A \cong A(1),[I, A]=Z(L)=Z(I)=K^{2}$ and $n=2 m+7$. Moreover, $I \rtimes A \cong\left\langle x_{1}, \ldots, x_{6}, x_{7}\right|\left[x_{1}, x_{2}\right]=x_{3},\left[x_{1}, x_{3}\right]=\left[x_{2}, x_{4}\right]=x_{5},\left[x_{1}, x_{5}\right]=$ $\left.\left[x_{3}, x_{4}\right]=\left[x_{2}, x_{7}\right]=\left[x_{4}, x_{7}\right]=x_{6}\right\rangle$.

Proof. It is similar to the proof of Theorem 3.4.

$\mathrm{W}$ are ready to determine the central factors of all stem Lie algebras $T$ such that $c l(T)=4$ and $\operatorname{dim} T^{2}=3$.

Lemma 3.6. Let $T$ be an $n$-dimensional stem Lie algebra such that $\operatorname{cl}(T)=4$ and $\operatorname{dim} T^{2}=3$. Then $T / Z(T) \cong L_{4,3} \oplus A(n-4)$ or $T / Z(T) \cong L_{5,5} \oplus A(n-5)$.

Proof. By using Proposition 3.3, we have $T^{2} / Z(T) \cong A(2)$. Since $T / Z(T)$ is capable, Theorem 2.4 implies that $T / Z(T) \cong L_{4,3} \oplus A(n-4)$ or $T / Z(T) \cong L_{5,5} \oplus A(n-5)$. It completes the proof.

In the following theorem, we determine the structure of all stem Lie algebras of class 4 with the derived subalgebra of dimension 3 . 
Theorem 3.7. Let $T$ be an $n$-dimensional stem Lie algebra such that $c l(T)=4$ and $\operatorname{dim} T^{2}=3$. Then $T$ is isomorphic to one of the following Lie algebras.

(a) $T \cong L_{5,6}$.

(b) $T \cong L_{5,7}$

(c) $T \cong I \rtimes K \cong L_{6,11}$, where $I \cong L_{5,6}, K \cong A(1),[I, K]=Z(I)=Z(T)$ and $Z_{2}(T)=Z_{2}(I) \rtimes K$.

(d) $T \cong I \rtimes K \cong L_{6,12}$, where $I \cong L_{5,7}, K \cong A(1),[I, K]=Z(I)=Z(T)$ and $Z_{2}(T)=Z_{2}(I) \rtimes K$.

(e) $T \cong I_{1}+I_{2} \cong\left\langle x_{1}, \ldots, x_{5}, a, b\right|\left[x_{1}, x_{i}\right]=x_{i+1},\left[x_{2}, x_{3}\right]=[a, b]=x_{5}, 1 \leq i \leq$ $4\rangle$, where $I_{1} \cong L_{5,6}$ and $I_{2} \cong H(1)$.

(f) $T \cong I_{1} \dot{+} I_{2} \cong\left\langle x_{1}, \ldots, x_{5}, a, b \mid\left[x_{1}, x_{i}\right]=x_{i+1},[a, b]=x_{5}, 1 \leq i \leq 4\right\rangle$, where $I_{1} \cong L_{5,7}$ and $I_{2} \cong H(1)$.

(g) $T \cong I_{1}+K_{1}$, where $I_{1} \cong\left\langle x_{1}, \ldots, x_{5}, a, b\right|\left[x_{1}, x_{i}\right]=x_{i+1},\left[x_{2}, x_{3}\right]=[a, b]=$ $\left.x_{5}, 1 \leq i \leq 4\right\rangle, K_{1} \cong H(m-1)$ and $n=2 m+5$ for all $m \geq 2$.

(h) $T \cong I_{2}+K_{2}$, where $I_{2} \cong\left\langle x_{1}, \ldots, x_{5}, a, b\right|\left[x_{1}, x_{i}\right]=x_{i+1},[a, b]=x_{5}, 1 \leq i \leq$ $4\rangle, K_{2} \cong H(m-1)$ and $n=2 m+5$ for all $m \geq 2$.

(k) $T \cong(I \rtimes K)+I_{3}$, where $I_{3} \cong H(m), K \cong A(1),[I, K]=Z(I)=Z(T)$, $I \cong L_{5,6}, I \rtimes K \cong L_{6,11}, Z_{2}(T)=\left(Z_{2}(I) \rtimes K\right)+I_{3}$ and $n=2 m+6$.

(l) $T \cong(I \rtimes K) \dot{+} I_{4}$, where $I_{4} \cong H(m), K \cong A(1),[I, K]=Z(I)=Z(T)$, $I \cong L_{5,7}, I \rtimes K \cong L_{6,12}, Z_{2}(T)=\left(Z_{2}(I) \rtimes K\right)+I_{4}$ and $n=2 m+6$.

(m) $T \cong L_{6,13}$.

(n) $T \cong\left\langle x_{1}, \ldots, x_{6}, x_{7}\right|\left[x_{1}, x_{2}\right]=x_{3},\left[x_{1}, x_{3}\right]=\left[x_{2}, x_{4}\right]=x_{5},\left[x_{1}, x_{5}\right]=\left[x_{3}, x_{4}\right]=$ $\left.\left[x_{2}, x_{7}\right]=\left[x_{4}, x_{7}\right]=x_{6}\right\rangle$.

(p) $T=I_{5}+K \cong\left\langle x_{1}, \ldots, x_{6}, a, b\right|\left[x_{1}, x_{2}\right]=x_{3},\left[x_{1}, x_{3}\right]=\left[x_{2}, x_{4}\right]=x_{5},\left[x_{1}, x_{5}\right]=$ $\left.\left[x_{3}, x_{4}\right]=[a, b]=x_{6}\right\rangle$, where $I_{5} \cong L_{6,13}$ and $K \cong H(1)$.

(q) $T \cong I_{6}+I_{7}$, where $I_{6} \cong\left\langle x_{1}, \ldots, x_{6}, a, b\right|\left[x_{1}, x_{2}\right]=x_{3},\left[x_{1}, x_{3}\right]=\left[x_{2}, x_{4}\right]=$ $\left.x_{5},\left[x_{1}, x_{5}\right]=\left[x_{3}, x_{4}\right]=[a, b]=x_{6}\right\rangle$ and $I_{7} \cong H(m-1)$ for all $m \geq 2$.

(r) $T \cong\left(I_{8} \rtimes A\right)+K_{3}$, where $K_{3} \cong H(m), I_{8} \cong L_{6,13}, A \cong A(1),\left[I_{8}, A\right]=$ $Z\left(I_{8}\right)=K^{2}$ and $n=2 m+7$. Moreover, $I_{8} \rtimes A \cong\left\langle x_{1}, \ldots, x_{7}\right|\left[x_{1}, x_{2}\right]=$ $\left.x_{3},\left[x_{1}, x_{3}\right]=\left[x_{2}, x_{4}\right]=x_{5},\left[x_{1}, x_{5}\right]=\left[x_{3}, x_{4}\right]=\left[x_{2}, x_{7}\right]=\left[x_{4}, x_{7}\right]=x_{6}\right\rangle$.

Proof. Since $\operatorname{cl}(T)=4$, we have $\operatorname{dim} T \geq 5$. If $\operatorname{dim} T=5$, then $T$ is a 5 -dimensional Lie algebra of maximal class and hence $T \cong L_{5,6}$ or $T \cong L_{5,7}$.

Assume that $\operatorname{dim} T \geq 6$. Thus $\operatorname{dim} T / Z(T) \geq 5$ and so $\operatorname{dim} I_{2} \geq 2$. Proposition 3.3 implies $T / Z(T) \cong L_{4,3} \oplus A(n-4)$ or $T / Z(T) \cong L_{5,5} \oplus A(n-5)$ and $Z(T)=$ $T^{4} \cong A(1)$. First let $T / Z(T) \cong L_{4,3} \oplus A(n-4)$. There exist two ideals $I_{1} / Z(T)$ and $I_{2} / Z(T)$ of $T / Z(T)$ such that $I_{1} / Z(T) \cong L_{4,3}$ and ${ }_{2} / Z(T) \cong A(n-5)$. Since $T^{2} / Z(T)=\left(I_{1}^{2}+Z(T)\right) / Z(T)$, we have $T^{2}=I_{1}^{2}+Z(T)$ and $Z(T)=T^{4}$. Using Lemma 2.7 we have $T^{2}=I_{1}^{2}$ and so $c l(T)=\operatorname{cl}\left(I_{1}\right)=4$. Hence $I_{1}$ is a 5 -dimensional Lie algebra of maximal class and so $I_{1} \cong L_{5,6}$ or $I_{1} \cong L_{5,7}$. Now, $Z(T)=Z\left(I_{1}\right)$ because $Z(T) \cap I_{1} \subseteq Z\left(I_{1}\right)$ and $\operatorname{dim} Z(T)=1$. Since $Z(T) \subseteq I_{1} \cap I_{2} \subseteq Z(T)$, we have $I_{1} \cap I_{2}=Z(T)=Z\left(I_{1}\right)$. Hence

$$
Z_{2}(T) / Z(T)=Z(T / Z(T))=Z\left(I_{1} / Z(T)\right) \oplus I_{2} / Z(T)=Z_{2}\left(I_{1}\right) / Z(T)+I_{2} / Z(T) .
$$

Since $I_{1}$ is maximal class of dimension 5 , we have $Z_{2}(T)=Z_{2}\left(I_{1}\right)+I_{2}=I_{1}^{3}+I_{2}$. Now, we are going to determine the structure of $I_{2}$. Since $I_{2} / Z(T) \cong A(n-5)$, we have $I_{2}^{2} \subseteq Z(T) \cong A(1)$ and hence $\operatorname{cl}\left(I_{2}\right) \leq 2$. Let $\operatorname{cl}\left(I_{2}\right)=1$. Therefore $\left[I_{1}, I_{2}\right]=I_{1} \cap I_{2}=Z(T)=Z\left(I_{1}\right) \cong A(1)$. Otherwise $\left[I_{1}, I_{2}\right]=0$ and since $I_{2}$ is abelian, $I_{2} \subseteq Z(T) \cong A(1)$. It is a contradiction, since $\operatorname{dim} I_{2} \geq 2$. Hence 
$I_{2}=Z(T) \oplus A$ in which $A \cong A(n-5)$ and $\left[I_{1}, I_{2}\right]=Z(T)$. Now $Z(T) \subseteq I_{1}, A \cap I_{1}=0$ and $I_{1} \cap I_{2}=Z(T)$ so $T=I_{1}+I_{2}=I_{1}+Z(T)+A=I_{1} \rtimes A$. Using the proof of Theorem $3.4(i)$, we have $T \cong I_{1} \rtimes K \cong L_{6,11}$ in which $K \cong A(1)$ and $\left[K, I_{1}\right]=Z(T)$ or $T \cong I_{1} \rtimes K \cong L_{6,12}$ in which $K \cong A(1)$ and $\left[K, I_{1}\right]=Z(T)$.

Now, let $\operatorname{cl}\left(I_{2}\right)=2$. Since $I_{2}^{2}=I_{1} \cap I_{2}=Z(T)=Z\left(I_{1}\right) \cong A(1)$, 11, Theorem 3.6] implies $I_{2} \cong H(m) \oplus A(n-2 m-5)$. First, assume that $A(n-2 m-5)=0$. Then $n=2 m+5$ and $I_{2} \cong H(m)$. Using Theorem 3.5 (ii), we can similarly obtain that $\left[I_{1}, I_{2}\right]=0$ and $T=I_{1} \dot{+} I_{2}$, where $I_{2} \cong H(m)$. This is cases $(e),(f),(g)$ and $(h)$. If $A(n-2 m-5) \neq 0$, then $n \neq 2 m+5$ and hence $T=I_{1}+(K \oplus A)$ where $K \cong H(m)$ and $A \cong A(n-2 m-5)$ and $\left[I_{1}, K \oplus A\right] \subseteq Z(T)=Z\left(I_{1}\right)$. Using Theorem3.5 (ii), we have $\left[I_{1}, K\right]=0$. Now, we claim that $\left[I_{1}, A\right]=Z(T) \cong A(1)$. Let $\left[I_{1}, A\right]=0$. Since $[K, A]=0$, we have $A \subseteq Z(T)=Z\left(I_{1}\right)=Z(K)=K^{2} \cong A(1)$. It is a contradiction, since $A \cap K=0$. Therefore $\left[I_{1}, A\right]=Z(T)$ and hence $T \cong\left(I_{1} \rtimes A\right) \dot{+} K$ where $A \cong A(n-2 m-5)$ and $\left[I_{1}, A\right]=Z(T)=Z\left(I_{1}\right)$. Similar to cases $(c)$ and $(d)$, one can obtain $A \cong A(1)$, so $n=2 m+6$ and $\left[I_{1}, A\right]=Z(T)$. So $T=\left(I_{1} \rtimes A\right) \dot{+} K$ in which $A \cong A(1)$ and $\left[I_{1}, A\right]=Z(T)$. This is cases $(k)$ and $(l)$.

Now let $T / Z(T) \cong L_{5,5} \oplus A(n-5)$. Similarly, we can obtain all cases $(m),(n),(p)$, $(q)$ and $(r)$, by using Proposition 3.5. The result follows.

The capable stem Lie algebras of class 4 with the derived subalgebra of dimension 3 are characterized in the following.

Lemma 3.8. Let $L$ be an $n$-dimensional stem Lie algebra such that $c l(L)=4$ and $\operatorname{dim} L^{2}=3$. If $L$ is isomorphic to one of the Lie algebras $L \cong L_{5,6}, L \cong L_{5,7}$, $L \cong L_{6,11}, L \cong L_{6,12}$ or $L \cong L_{6,13}$, then $L$ is capable.

Proof. By using a terminology of [4, 7, we have $L_{6,15}=\left\langle x_{1}, \ldots, x_{6}\right|\left[x_{1}, x_{2}\right]=$ $\left.x_{3},\left[x_{1}, x_{3}\right]=x_{4},\left[x_{1}, x_{4}\right]=x_{5}=\left[x_{2}, x_{3}\right],\left[x_{1}, x_{5}\right]=x_{6}=\left[x_{2}, x_{4}\right]\right\rangle$ and $L_{6,18}=$ $\left\langle x_{1}, \ldots, x_{6} \mid\left[x_{1}, x_{2}\right]=x_{3},\left[x_{1}, x_{3}\right]=x_{4},\left[x_{1}, x_{4}\right]=x_{5},\left[x_{1}, x_{5}\right]=x_{6}\right\rangle$. It is clear to see that $Z\left(L_{6,15}\right)=\left\langle x_{6}\right\rangle$ and $Z\left(L_{6,18}\right)=\left\langle x_{6}\right\rangle$, so $L_{6,15} /\left\langle x_{6}\right\rangle \cong L_{5,6}$ and $L_{6,18} /\left\langle x_{6}\right\rangle \cong$ $L_{5,7}$. Hence they are capable. Consider $H=\left\langle x_{1}, \ldots, x_{7}\right|\left[x_{1}, x_{2}\right]=x_{3},\left[x_{1}, x_{3}\right]=$ $\left.x_{4}=\left[x_{2}, x_{7}\right],\left[x_{1}, x_{4}\right]=x_{5}=\left[x_{3}, x_{7}\right],\left[x_{1}, x_{5}\right]=x_{6}=\left[x_{4}, x_{7}\right]\right\rangle$. Clearly, $Z(H)=$ $\left\langle x_{6}\right\rangle$. Hence $L_{6,13} \cong H /\left\langle x_{6}\right\rangle$. Therefore $L_{6,13}$ is capable. The capability of $L_{6,11}$ and $L_{6,12}$ are obtained by a similar way. Hence the proof is completed.

The following lemma is a useful instrument in the next investigations.

Lemma 3.9. The Lie algebra $L=\left\langle x_{1}, x_{2}, \ldots, x_{6}, z\right|\left[x_{1}, x_{i}\right]=x_{i+1},\left[x_{2}, x_{6}\right]=$ $\left.x_{4},\left[x_{3}, x_{6}\right]=\left[x_{2}, z\right]=\left[x_{6}, z\right]=x_{5}, 1 \leq i \leq 4\right\rangle$ is non-capable.

Proof. Clearly, $Z(L)=\left\langle x_{5}\right\rangle$. We claim that $x_{5} \in Z^{\wedge}(L)$. It is sufficient to see that $x_{i} \wedge x_{5}=z \wedge x_{5}=0_{L \wedge L}$ for all $i, 1 \leq i \leq 6$. Since

$$
\begin{aligned}
& x_{1} \wedge x_{5}=x_{1} \wedge\left[x_{6}, z\right]=\left[z, x_{1}\right] \wedge x_{6}-\left[x_{6}, x_{1}\right] \wedge z=0_{L \wedge L}, \\
& x_{3} \wedge x_{5}=\left[x_{1}, x_{2}\right] \wedge x_{5}=x_{1} \wedge\left[x_{2}, x_{5}\right]-x_{2} \wedge\left[x_{1}, x_{5}\right]=0_{L \wedge L}, \\
& x_{4} \wedge x_{5}=\left[x_{1}, x_{3}\right] \wedge x_{5}=x_{1} \wedge\left[x_{3}, x_{5}\right]-x_{3} \wedge\left[x_{1}, x_{5}\right]=0_{L \wedge L}, \\
& x_{6} \wedge x_{5}=x_{6} \wedge\left[x_{1}, x_{4}\right]=\left[x_{4}, x_{6}\right] \wedge x_{1}-\left[x_{1}, x_{6}\right] \wedge x_{4}=0_{L \wedge L}, \\
& z \wedge x_{5}=z \wedge\left[x_{1}, x_{4}\right]=\left[x_{4}, z\right] \wedge x_{1}-\left[x_{1}, z\right] \wedge x_{4}=0_{L \wedge L}
\end{aligned}
$$


and

$$
\begin{aligned}
& x_{2} \wedge x_{5}=x_{2} \wedge\left[x_{6}, z\right]=\left[z, x_{2}\right] \wedge x_{6}-\left[x_{6}, x_{2}\right] \wedge z=-x_{5} \wedge x_{6}- \\
& {\left[x_{6}, x_{2}\right] \wedge z=-x_{4} \wedge z=-\left[x_{1}, x_{3}\right] \wedge z=-x_{1} \wedge\left[x_{3}, z\right]+x_{3} \wedge\left[x_{1}, z\right]=0_{L \wedge L},}
\end{aligned}
$$

$x_{5} \in Z^{\wedge}(L)$, as required.

Recall that a Lie algebra $L$ is called unicentral if $Z^{*}(L)=Z(L)$.

Theorem 3.10. Let $T$ be an $n$-dimensional stem Lie algebra such that $c l(T)=4$ and $\operatorname{dim} T^{2}=3$. Then $T$ is non-capable if and only if $n \geq 7$. Moreover, $T$ is unicentral.

Proof. Let $T$ be non-capable. Then Theorem 3.7 and Lemma 3.8 imply $n \geq 7$. Conversely, let $n \geq 7$. Then by Proposition 2.3. Theorem 3.7 and Lemma 3.9, $T$ is non-capable. The result follows.

In the following theorem, all capable stem Lie algebras of class 4 with the derived subalgebra of dimension 3 are given.

Theorem 3.11. Let $T$ be an $n$-dimensional stem Lie algebra such that $c l(T)=4$ and $\operatorname{dim} T^{2}=3$. Then $T$ is capable if and only if $T$ is isomorphic to one of the Lie algebras $L_{5,6}, L_{5,7}, L_{6,11}, L_{6,12}$ or $L_{6,13}$.

Proof. Let $T$ be capable. By Theorem 3.7, Lemma 3.8 and Theorem 3.10, $T$ is isomorphic to one of the Lie algebras $L_{5,6}, L_{5,7}, L_{6,11}, L_{6,12}$ or $L_{6,13}$. The converse holds by Lemma 3.8 .

The next theorem gives a necessary and sufficient condition for detecting the capability of stem Lie algebras of class 4 with the derived subalgebra of dimension 3.

Theorem 3.12. Let $T$ be an $n$-dimensional stem Lie algebra such that $c l(T)=4$ and $\operatorname{dim} T^{2}=3$. Then $T$ is capable if and only if $4 \leq \operatorname{dim}(T / Z(T)) \leq 5$.

Proof. The result follows from Lemma 3.6 and Theorem 3.11

The following result shows that each finite dimensional nilpotent Lie algebra $L$ of class 4 with the derived subalgebra of dimension 3 has a decomposition into a stem Lie algebra $T$ of class 4 with $\operatorname{dim} T^{2}=3$ and an abelian Lie algebra. Moreover, there is a nice relationship between the capability of $L$ and $T$.

Theorem 3.13. Let $L$ be a finite dimensional nilpotent Lie algebra of class 4 and $\operatorname{dim} L^{2}=3$. Then $L=T \oplus A$ such that $Z(T)=L^{2} \cap Z(L)=L^{4}=T^{4}$ and $Z^{*}(L)=Z^{*}(T)$, where $A$ is an abelian Lie algebra.

Proof. By using [8, Proposition 3.1], $L=T \oplus A$ such that $Z(T)=L^{2} \cap Z(L)$ and $Z^{*}(L)=Z^{*}(T)$, where $A$ is an abelian Lie algebra. Since $T$ is stem, Proposition 3.3 implies $Z(T)=T^{4}$, as required.

Now, we are in the position to determine all capable Lie algebras $L$ of class 4 whit $\operatorname{dim} L^{2}=3$.

Theorem 3.14. Let $L$ be an $n$-dimensional Lie algebra such that $\operatorname{cl}(L)=4$ and $\operatorname{dim} L^{2}=3$. Then $L$ is capable if and only if $L$ is isomorphic to one of the Lie algebras $L_{5,6} \oplus A(n-5), L_{5,7} \oplus A(n-5), L_{6,11} \oplus A(n-6), L_{6,12} \oplus A(n-6)$ or $L_{6,13} \oplus A(n-6)$. 
Proof. By using Theorem 3.13, $L=T \oplus A$ such that $Z(T)=L^{2} \cap Z(L)=L^{4}=$ $T^{4} \cong A(1)$ and $Z^{*}(L)=Z^{*}(T)$, where $A$ is an abelian Lie algebra. Now, the result follows from Theorem 3.11

The following result is obtained from Theorems 3.12 and 3.14

Corollary 3.15. Let $L$ be a finite dimensional Lie algebra of class 4 and $\operatorname{dim} L^{2}=$ 3. Then $L$ is capable if and only if $4 \leq \operatorname{dim}(L / Z(L)) \leq 5$.

\section{REFERENCES}

[1] F. R. Beyl, U. Felgner and P. Schmid, On groups occurring as center factor groups, J. Algebra 61 (1970) 161-177.

[2] N. Blackburn, On a special class of p-groups, Acta Math. 100 (1958), no. 1-2, 45-92.

[3] L. Bosko, On Schur multiplier of Lie algebras and groups of maximal class, Internat. J. Algebra Comput. 20 (2010) 807-821.

[4] S. Cical, W. A. de Graaf and C. Schneider, Six-dimensional nilpotent Lie algebras, Linear Algebra Appl. 436 (2012), no. 1, 163-189.

[5] G. Ellis, A non-abelian tensor product of Lie algebras, Glasg. Math. J. 39 (1991) 101-120.

[6] M. P. Gong, Classification of nilpotent Lie Algebras of dimension 7 (over Algebraically closed fields and R), A thesis in Waterloo, Ontario, Canada, 1998.

[7] W. A. de Graaf, Classification of 6-dimensional nilpotent Lie algebras over fields of characteristic not 2, J. Algebra 309 (2007) 640-653.

[8] F. Johari, M. Parvizi and P. Niroomand, Capability and Schur multiplier of a pair of Lie algebras, J. Geom. Phys. 114 (2017), 184-196

[9] K. Moneyhun, Isoclinisms in Lie algebras, Algebras Groups Geom. 11 (1994), no.1, 9-22.

[10] P. Niroomand, On the dimension of the Schur multiplier of nilpotent Lie algebras, Cent. Eur. J. Math. 9 (2011) 57-64.

[11] P. Niroomand, M. Parvizi and F. G. Russo, Some criteria for detecting capable Lie algebras, J. Algebra 384 (2013) 36-44.

[12] P. Niroomand, F. Johari and M. Parvizi, On the capability and Schur multiplier of nilpotent Lie algebra of class two, Proc. Amer. Math. Soc. 144 (2016), no. 10, 4157-4168.

[13] P. Niroomand, F. Johari and M. Parvizi, Capable Lie algebras with the derived subalgebra of dimension two over an arbitrary filed, Linear and Multilinear Algebra, DOI: 10.1080/03081087.2018.1425356.

[14] A. R. Salemkar, V. Alamian and H. Mohammadzadeh, Some properties of the Schur multiplier and covers of Lie Algebras, Comm. Algebra 36 (2008) 697-707.

[15] L. M. Zack, Nilpotent Lie algebras with a small second derived quotient, Comm. Algebra 36 (2008), no. 12, 4607-4619.

Department of Pure Mathematics, Ferdowsi University of Mashhad, Mashhad, Iran E-mail address: farangisjohary@yahoo.com, farangis.johari@mail.um.ac.ir

School of Mathematics and Computer Science, Damghan University, Damghan, Iran

E-mail address: niroomand@du.ac.ir, p_niroomand@yahoo.com

Department of Pure Mathematics, Ferdowsi University of Mashhad, Mashhad, Iran

E-mail address: parvizi@math.um.ac.ir 